OPEN ACCESS

Edited by:

Wen-Lung Ma,

China Medical University, Taiwan

Reviewed by:

Nancy Kemeny,

Memorial Sloan Kettering Cancer

Center, United States

Graeme Poston,

University of Liverpool, United

Kingdom

${ }^{*}$ Correspondence:

Jia Wu

tkds123@qq.com

Yuhua Zhang

drzhangyuhua@126.com

†These authors have contributed equally to this work and share

first authorship

*These authors have contributed equally to this work and share

last authorship

Specialty section: This article was submitted to Gastrointestinal Cancers: Hepato

Pancreatic Biliary Cancers, a section of the journal

Frontiers in Oncology

Received: 14 August 2021 Accepted: 06 October 2021 Published: 22 October 2021

Citation:

Chen Y, Xu Y, Xu L, Han F, Huang Y,

Jiang $H, W u J$ and Zhang $Y(2021)$ Neoadjuvant Chemotherapy Followed by Radiofrequency Ablation Prolongs Survival for Ablatable Colorectal Liver Metastasis: A Propensity Score Matching Comparative Study.

Front. Oncol. 11:758552. doi: 10.3389/fonc.2021.758552

\section{Neoadjuvant Chemotherapy Followed by Radiofrequency Ablation Prolongs Survival for Ablatable Colorectal Liver Metastasis: A Propensity Score Matching Comparative Study}

\author{
Yizhen Chen ${ }^{1,2 \dagger}$, Youyao $X u^{1,2 \dagger}$, Linwei $X u^{1}$, Fang Han $^{1}$, Yurun Huang ${ }^{1,2}$, Hang Jiang ${ }^{1,2}$ \\ Jia Wu ${ }^{1 * \neq}$ and Yuhua Zhang ${ }^{1 * \neq}$ \\ ${ }^{1}$ Department of Hepatobiliary and Pancreatic Surgery, The Cancer Hospital of the University of Chinese Academy of \\ Sciences (Zhejiang Cancer Hospital), Institute of Basic Medicine and Cancer (IBMC), Chinese Academy of Sciences, \\ Hangzhou, China, ${ }^{2}$ Zhejiang Chinese Medical University, Hangzhou, China
}

Background: Typically, colorectal liver metastasis (CRLM) is not a candidate for hepatectomy. Radiofrequency ablation (RFA) plays a critical role in unresectable CRLM patients. Nevertheless, high local tumor progression (LTP) and distant metastasis limit the development and further adoption and use of RFA. Neoadjuvant chemotherapy (NAC) has been widely used in resectable CRLM and is recommended by the guidelines. There are no studies on whether NAC can improve the prognosis in ablatable CRLM patients. The present study aimed to determine the feasibility and effectiveness of RFA plus NAC.

Methods: This retrospective cohort included CRLM patients from Zhejiang Cancer Hospital records, who received RFA from January 2009 to June 2020 and were divided into two groups according to the presence or absence of NAC. The KaplanMeier method was used to evaluate the 3-year local tumor progression-free survival (LTPFS), progression-free survival (PFS), and overall survival (OS) of the two groups. The propensity score matching was used to reduce bias when assessing survival. Multivariate Cox proportional hazards regression analysis was used to study the independent factors affecting LTPFS, PFS, and OS.

Results: A total of 149 CRLM patients (88 in the RFA alone group and 61 in the plus NAC group) fulfilled the inclusion criteria. Post-RFA complications were $3.4 \%$ in the RFA alone group and $16.4 \%$ in the plus NAC group. The 3-year LTPFS, PFS, and OS of the RFA only group were $60.9 \%, 17.7 \%$, and $46.2 \%$, respectively. The 3-year LTPF, PFS, and OS of the plus NAC group were $84.9 \%, 46.0 \%$, and $73.6 \%$, respectively. In the 29 pairs of propensity score matching cohorts, the 3-year LTPFS, PFS, and OS in the plus NAC group were longer than those in the RFA group $(P<0.05)$. NAC was an independent protective factor for LTPFS, PFS, and OS $(P<0.05)$. 
Conclusions: For ablatable CRLM patients, RFA plus NAC obtained a better prognosis than RFA alone. Based on the current results, the application of NAC before RFA may become the standard treatment.

Keywords: colorectal cancer, liver metastasis, neoadjuvant chemotherapy (NAC), radiofrequency ablation, local tumor progression (LTP)

\section{INTRODUCTION}

According to recent cancer statistics, colorectal cancer (CRC) is one of the most common cancers and the leading cause of cancer-related deaths worldwide $(1,2)$. Liver metastases are the most common site of distal spread in colorectal cancer (3). About $50 \%$ of CRC patients showed liver metastasis, which severely worsens the overall prognosis of CRC patients $(4,5)$.

For colorectal liver metastasis (CRLM), the purpose of the treatment is to improve progression-free survival (PFS) and overall survival (OS). Surgical resection is the preferred treatment for CRLM (6). Although the indications for hepatectomy have been expanding, about $80 \%$ of CRLM patients are not candidates for hepatectomy (7). Local ablation therapy represented by radiofrequency ablation (RFA) plays a critical role in CRLM patients (8). Although RFA has shown a prognosis similar to hepatectomy in some specific patients (9), the high rate of local tumor progression (LTP) is still an obstacle to using ablation therapy (10).

A significant benefit was obtained in PFS in resectable CRLM patients who underwent surgery after neoadjuvant chemotherapy (NAC) compared with the surgery alone (11). NAC has been routinely used in clinical practice and recommended by guidelines and consensus (12-14). For ablatable CRLM patients, whether NAC followed by RFA can achieve a better prognosis than RFA alone is currently under intensive focus. Therefore, the present study aimed to explore whether NAC can improve the prognosis in ablatable CRLM patients.

\section{MATERIALS AND METHODS}

\section{Study Population}

We conducted a retrospective review of all cases of ultrasound (US)-guided percutaneous RFA for CRLM in the Cancer Hospital of the University of Chinese Academy of Sciences (Zhejiang Cancer Hospital) from January 2009 to June 2020. All cases were confirmed pathologically as CRLM by liver biopsy during treatment. In a patient with multiple metastases, each metastasis was separately biopsied. The imaging data of these cases (before NAC or RFA) were re-evaluated by a multidisciplinary team composed of surgeons and interventional radiologists.

All initially ablatable CRLM patients undergoing RFA as the primary method of treatment for CLRM were included in this study. Combining the RFA guidelines and expert consensus (15), our center defined percutaneous ablatable criteria as the largest lesion diameter $\leq 5 \mathrm{~cm}$, and a maximum of five lesions could be ablated simultaneously. The lesion was far away from large blood vessels and major biliary tract, and sufficient liver was remnant after RFA (normal liver $\geq 20 \%$, after chemotherapy $\geq 30 \%$ ). The primary sites of these cases had been radically resected. All the cases were ablated completely, and the ablation margins were $>10 \mathrm{~mm}$. Patients who met any of the following criteria were excluded: 1) the MDT team determined that the patient was initially not suitable for RFA first; 2) extrahepatic metastasis; 3) prior to RFA, other treatments, such as liver resection and hepatic arterial infusion chemotherapy (except NAC), were performed; 4) severe dysfunction of vital organs; and 5) the follow-up time was $<6$ months.

The eligible patients were divided automatically into two groups for comparison: patients who had not received any treatment before RFA and those who received NAC before RFA. The primary endpoints of this study are LTP-free survival (LTPFS). Secondary endpoints included PFS and OS. This study was approved by the Medical Ethics Committee of the Cancer Hospital of the University of Chinese Academy of Sciences (Zhejiang Cancer Hospital). All patients provided written informed consent. In this study, the first choice for patients in the NAC group was not ablation (but surgical resection or palliative chemotherapy alone). After chemotherapy, the patient and the doctor negotiated to choose RFA for various reasons (for example, the preference of the patient and not suitable for surgery after chemotherapy).

\section{RFA Procedure}

RFA was carried out in collaboration with an interventional radiologist and a hepatobiliary surgeon. The interventional radiologist at our center had $>10$ years of experience in percutaneous liver ultrasonography and RFA. Percutaneous RFA was performed under general anesthesia. A 16-G bipolar electrode needle was used for RFA. The electrodes were placed in the lesion under US guidance. RFA was performed three times for each lesion site until the rolling endpoint was reached. Consequently, $>1 \mathrm{~cm}$ ablation margin was achieved. For lesions $>3 \mathrm{~cm}$, multiple overlapping ablations were required along with continuous monitoring of local temperature and tissue impedance. During RFA, US in the ablation zone showed high echo, encompassing the lesion area. Contrastenhanced CT was conducted immediately following the procedure in all cases to assess whether the ablation is complete and no residual viable tumor remained within the ablation zone.

\section{Definitions}

RFA effectiveness is defined as the ablation defect completely surrounding the targeted tumor, and the failure is defined as 
evidence of residual tumor within $1 \mathrm{~cm}$ of the ablation defect. NAC is defined as ablatable CRLM patients who underwent at least one cycle of chemotherapy before RFA. LTP is defined as any new peripheral/nodular enhancement or enlargement of ablation defect within $1 \mathrm{~cm}$ of the RFA area (16). LTPFS is defined as the duration interval between the first RFA and the occurrence of LTP. OS is defined as the duration from RFA to the date of death or to the date of the last follow-up. PFS is defined as the duration from RFA to the confirmation of recurrence or death. Objective response was evaluated by CT or MRI scans based on Response Evaluation Criteria in Solid Tumors (RECIST) version 1.1. The modified ablation clinical risk score (CRS) is as follows: node-positive primary tumor, disease-free interval from primary resection to the diagnosis of liver metastasis $<12$ months, more than one liver tumor, size of largest tumor $>3 \mathrm{~cm}$, and carcinoembryonic antigen (CEA) level $>30 \mathrm{ng} / \mathrm{ml}$ (17). One point was assigned to each item.

\section{Data Collection and Follow-Up}

The baseline characteristics, the course of the disease during RFA (such as complications), NAC regimens, and cycles were obtained from the electronic medical record system. The follow-up was up to May 30, 2021, to obtain the survival status of patients. Each patient was asked to return to the hospital every 3 months after RFA to confirm the progress of the disease by CT or contrast-enhanced ultrasound or MRI. The interval of the follow-up was set at 6 months if the patient was progression-free in the initial 2 years, and the frequency of follow-up was adjusted to once a year if the patients kept the status of progression-free over 5 years.

\section{Statistical Analysis}

All statistical analyses were performed using SPSS statistical software (version 25, SPSS Inc., Chicago, IL, USA). The Pearson's $\chi^{2}$ or Fisher exact test was used to compare the baseline characteristics of the two groups. The LTPFS, OS, and PFS of the two groups were estimated using the Kaplan-Meier method and compared by log-rank test. A Cox proportional hazard multiple regression model was established. First, the univariate analysis was conducted, and related factors $(P<0.1)$ were included in the multivariate analysis. In the multivariate analysis, factors with $P<0.05$ were considered as independent predictors of LTPPFS, OS, and PFS.

The independent factors related to LTPFS, PFS, and OS and baseline characteristics with significant differences are CEA at diagnosis, timing of metastasis, number of liver metastases, largest diameter, adjuvant chemotherapy after RFA, and modified ablation CRS. The two groups were then formed using a one-to-one nearest neighbor caliper with a width of 0.03 .

\section{RESULTS}

\section{Clinicopathological Characteristics}

From 2009 to 2020, a total of 149 CRLM patients met the inclusion criteria: 88 cases comprised the RFA group alone, and 61 cases were in the plus NAC group. The mean age of all patients is $59.1 \pm 12.3$ years (standard deviation), and the ratio of males to females in the total cohort is 96:53. The clinical characteristics of the two groups of patients are summarized in Table 1. Except for age, the timing of liver metastases, number of liver metastases, adjuvant chemotherapy after RFA, and modified ablation CRS, the other baseline characteristics of the two groups did not differ significantly. Nearly half of the patients $(49.2 \%)$ in the group receiving NAC achieved an objective response. In the plus NAC group, 40/61 patients used the XELOX regimen, 10/61 patients used the FOLFOX regimen, and 11/61 patients used the FOLFIRI regimen. Moreover, 13/61 patients in the plus NAC group were combined with targeted drugs ( 7 cases of cetuximab and 6 cases of bevacizumab). The median number of cycles of NAC is $4(3,6)$. After propensity score matching, we obtained a one-to-one paired cohort of the plus NAC group and the RFA alone group (29 patients in each group) (Table 2). In the matched cohort, there were no longer any significant differences between two groups in any key confounding factors at baseline.

\section{Post-RFA Complications}

The analysis of complications after RFA is shown in Table 3. The postoperative complication rate of the plus NAC group was higher than that of the RFA alone group ( $16.4 \%$ vs. $3.4 \%)$.

TABLE 1 | Baseline characteristics of the patients.

\begin{tabular}{|c|c|c|c|}
\hline Variables & RFA alone & NAC & $P^{a}$ \\
\hline$N$ & 88 & 61 & \\
\hline \multicolumn{4}{|l|}{ Age } \\
\hline$\leq 60 />60$ & $40 / 48$ & $38 / 23$ & 0.043 \\
\hline \multicolumn{4}{|l|}{ Gender } \\
\hline Female/male & $26 / 62$ & $27 / 34$ & 0.065 \\
\hline \multicolumn{4}{|l|}{ CEA at diagnosis, ng/ml } \\
\hline$\leq 30 />30$ & $72 / 16$ & $44 / 17$ & 0.161 \\
\hline \multicolumn{4}{|l|}{ Location of primary cancer } \\
\hline Colon/rectum & $43 / 45$ & $26 / 35$ & 0.453 \\
\hline \multicolumn{4}{|l|}{ T stage of primary tumor } \\
\hline $\mathrm{T} 1-\mathrm{T} 2 / \mathrm{T} 3-\mathrm{T} 4$ & $8 / 80$ & $9 / 52$ & 0.285 \\
\hline \multicolumn{4}{|l|}{$\mathrm{N}$ stage of primary tumor } \\
\hline $\mathrm{NO} / \mathrm{N}_{+}$ & $24 / 64$ & $11 / 50$ & 0.191 \\
\hline \multicolumn{4}{|l|}{ Timing of metastasis } \\
\hline Metachronous/synchronous & $78 / 10$ & $23 / 38$ & $<0.001$ \\
\hline \multicolumn{4}{|l|}{ Number of liver metastases } \\
\hline$<3 / \geq 3$ & $82 / 6$ & $40 / 21$ & $<0.001$ \\
\hline \multicolumn{4}{|l|}{ Largest diameter (cm) } \\
\hline$<3 / \geq 3$ & $69 / 19$ & $41 / 20$ & 0.126 \\
\hline \multicolumn{4}{|c|}{ Adjuvant chemotherapy after RFA } \\
\hline No/yes & $63 / 25$ & $18 / 43$ & $<0.001$ \\
\hline \multicolumn{4}{|l|}{ Response to NAC } \\
\hline $\mathrm{CR}+\mathrm{PR} / \mathrm{SD}+\mathrm{PD}$ & & $30 / 31$ & \\
\hline \multicolumn{4}{|l|}{ Modified ablation $\mathrm{CRS}^{\mathrm{b}}$} \\
\hline $0-2 / 3-5$ & $73 / 15$ & $30 / 31$ & $<0.001$ \\
\hline
\end{tabular}

$C R$, complete response; $P R$, partial response; $S D$, stable disease; $P D$, progressive disease; CEA, carcinoembryonic antigen.

'The Pearson's $\chi^{2}$ or Fisher exact test was used to compare the basic characteristics of the two groups.

${ }^{b}$ Node-positive primary tumor + disease-free interval from primary resection to the diagnosis of liver metastasis $<12$ months + more than one liver tumor + size of largest tumor $>3 \mathrm{~cm}+$ CEA level $>30 \mathrm{ng} / \mathrm{ml}(\mathrm{mg} / \mathrm{L})$. 
TABLE 2 | Baseline characteristics of the patients after propensity score matching

\begin{tabular}{|c|c|c|c|}
\hline Variables & RFA alone & NAC & $P^{\mathrm{a}}$ \\
\hline N & 29 & 29 & \\
\hline \multicolumn{4}{|l|}{ Age } \\
\hline$\leq 60 />60$ & $16 / 13$ & 19/10 & 0.4 \\
\hline \multicolumn{4}{|l|}{ Gender } \\
\hline Female/male & $13 / 16$ & $12 / 17$ & 0.7 \\
\hline \multicolumn{4}{|l|}{ CEA at diagnosis, $\mathrm{ng} / \mathrm{ml}$} \\
\hline$\leq 30 />30$ & $21 / 8$ & $22 / 7$ & 0.7 \\
\hline \multicolumn{4}{|l|}{ Location of primary cancer } \\
\hline Colon/rectum & $12 / 17$ & $10 / 19$ & 0.5 \\
\hline \multicolumn{4}{|l|}{ T stage of primary tumor } \\
\hline T1-T2/T3-T4 & $1 / 28$ & $6 / 23$ & 0.10 \\
\hline \multicolumn{4}{|l|}{$\mathrm{N}$ stage of primary tumor } \\
\hline $\begin{array}{l}\mathrm{NO} / \mathrm{N}+ \\
\text { Timing of metastasis }\end{array}$ & $8 / 21$ & Timing of metastasis & 0.53 \\
\hline Metachronous/synchronous & $22 / 7$ & $22 / 7$ & 1.00 \\
\hline \multicolumn{4}{|l|}{ Number of liver metastases } \\
\hline$<3 / \geq 3$ & $25 / 4$ & $26 / 3$ & 1.00 \\
\hline \multicolumn{4}{|l|}{ Largest diameter (cm) } \\
\hline \multicolumn{3}{|c|}{ Adjuvant chemotherapy after RFA } & $0.7 \varepsilon$ \\
\hline \multicolumn{4}{|l|}{ Modified ablation $\mathrm{CRS}^{\mathrm{b}}$} \\
\hline $0-2 / 3-5$ & $22 / 7$ & $17 / 12$ & 0.16 \\
\hline
\end{tabular}

CEA, carcinoembryonic antigen.

${ }^{a}$ The Pearson's $\chi^{2}$ or Fisher exact test was used to compare the basic characteristics of the two groups.

${ }^{b}$ Node-positive primary tumor + disease-free interval from primary resection to the diagnosis of liver metastasis $<12$ months + more than one liver tumor + size of largest tumor $>3 \mathrm{~cm}+$ CEA level $>30 \mathrm{ng} / \mathrm{ml}$ ( $\mathrm{mg} / \mathrm{L})$.

The postoperative complications of NAC were mainly abdominal infection. Severe complications $(\mathrm{CD} \geq 3)$ occurred in only one case in the plus NAC group, and no severe complications were detected in the RFA alone group. No mortality was observed within 30 days after the RFA.

\section{Survival Analysis}

The median follow-up time for all patients was 30.0 months. As shown in Figures 1A, 2A, and 3A, the plus NAC group increased the 3 -year LTPFS ( $84.9 \%$ vs. $60.9 \%, P<0.001)$, PFS $(46.0 \%$ vs. $17.7 \%, P<0.001)$, and OS ( $73.6 \%$ vs. $46.2 \%, P=0.007)$ of CRLM patients compared with the RFA alone group. The 3-year LTP rate was $39.1 \%$ and $15.1 \%$ in the RFA alone and the plus NAC groups, respectively. The results showed that poor LTPFS was independently related to the size of the largest lesion $\geq 3 \mathrm{~cm}$ [hazard ratio (HR), 3.837; 95\% CI: 1.973-7.463; $P<0.001$ ], and

TABLE 3 | Post-RFA complications.

\begin{tabular}{lcc}
\hline Variables & RFA alone, $\boldsymbol{N}(\%)$ & NAC, $\boldsymbol{N}(\%)$ \\
\hline Overall complications & $3(3.4)$ & $10(16.4)$ \\
Abdominal bleeding & $1(1.1)$ & $0(0.0)$ \\
Abdominal infection & $1(1.1)$ & $7(11.5)$ \\
Liver failure & $1(1.1)$ & $2(3.3)$ \\
Pleural effusion & $0(0.0)$ & $1(1.7)$ \\
Serious complications $(C D \geq 3)$ & $0(0.0)$ & $1(1.7)$ \\
\hline
\end{tabular}

$C D$, Clavien-Dindo classification. improved LTPFS was independently related to the use of NAC (HR, 0.219; 95\% CI: 0.088-0.543; $P=0.001$ ) (Table 4). The findings revealed that better PFS is independently related to NAC (HR, 0.430; 95\% CI: 0.263-0.704; $P=0.001$ ) (Table 5). Also, enhanced OS is independently related to NAC (HR, 0.427; 95\% CI: $0.247,0.739 ; P=0.002)$ and adjuvant chemotherapy after RFA (HR, 0.540; 95\% CI: 0.323, 0.902; $P=0.019$ ) (Table 6). Also, OS is independently related to CEA $>30 \mathrm{ng} / \mathrm{ml}$ (HR, 1.840; 95\% CI: $1.045-3.239 ; P=0.035)$. After propensity score matching, the 3-year LTPFS, PFS, and OS of the plus NAC and RFA alone groups were significantly different $(96.6 \%$ vs. $71.4 \%$ in LTPFS, $P=0.024$, Figure $1 \mathbf{B} ; 58.7 \%$ vs. $21.7 \%$ in PFS, $P=0.009$, Figure $2 \mathrm{~B} ; 80.2 \%$ vs. $41.5 \%$ in OS, $P=0.041$, Figure 3B, respectively).

\section{DISCUSSION}

Liver metastasis worsens the prognosis of CRC patients. As a commonly used minimally invasive approach, RFA is characterized by less trauma, fewer complications, and a short hospital stay, which has been widely used in the local control of CRLM (18). For unresectable CRLM patients, RFA with postoperative chemotherapy achieved a better prognosis than chemotherapy alone $(19,20)$. However, compared with surgical resection, RFA for CRLM was considered to have a high recurrent rate, irrespective of being extrahepatic or intrahepatic, and to have a low survival rate (21). Therefore, for patients who need local treatment, prolonging their survival is crucial. Most of the current consensus definitions are as follows: I) unresectable liver lesions; II) combination with hepatectomy; III) patients with significant medical comorbidities or World Health Organization (WHO) poor performance status (performance status $>1$ ); IV) a small $(<3 \mathrm{~cm})$ solitary lesion, which would otherwise necessitate a major liver resection; and V) patient preference $(8,22)$. NAC has been widely used in the clinical treatment of CRLM and is recommended by guidelines and consensus (12-14). The multivariate analysis of retrospective studies suggested that NAC is an independent predictor of survival in resectable CRLM patients, which increases the probability of R0 and the remaining liver volume after surgical resection (23). In addition, NAC improves the prognosis of CRLM patients and prolongs survival (24). The guidelines and consensus were focused on NAC for resectable CRLM patients. However, no studies were associated to ablatable CRLM. The current study focused on investigating whether NAC followed by RFA could achieve a better prognosis than RFA alone for ablatable CRLM patients.

The 3-year LTPFS, PFS, and OS of the RFA only group were $60.9 \%, 17.7 \%$, and $46.2 \%$, respectively. Surprisingly, the two groups have significant differences in LTPFS, PFS, and OS, and the plus NAC group showed an improved prognostic advantage $(84.9 \%, 46.0 \%$, and $73.6 \%$, respectively). NAC was associated with LTPFS, PFS, and OS, which reduced the risk of LTP by $88 \%(\mathrm{HR}=0.219, P=0.001)$, the risk of systemic disease progression by $53 \%(\mathrm{HR}=0.430, P=0.001)$, and the risk of death by $53 \%(\mathrm{HR}=0.427, P=0.002)$. The credibility of these 
A

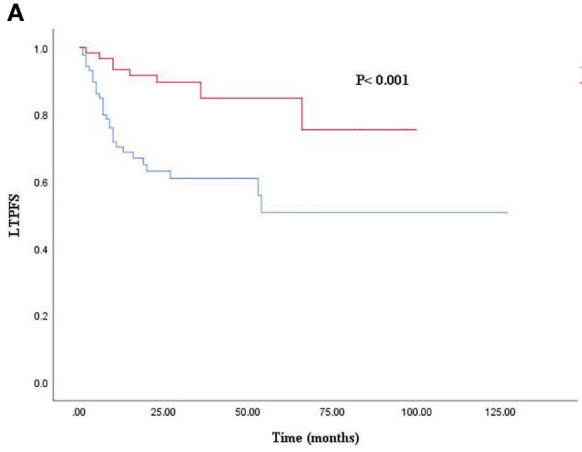

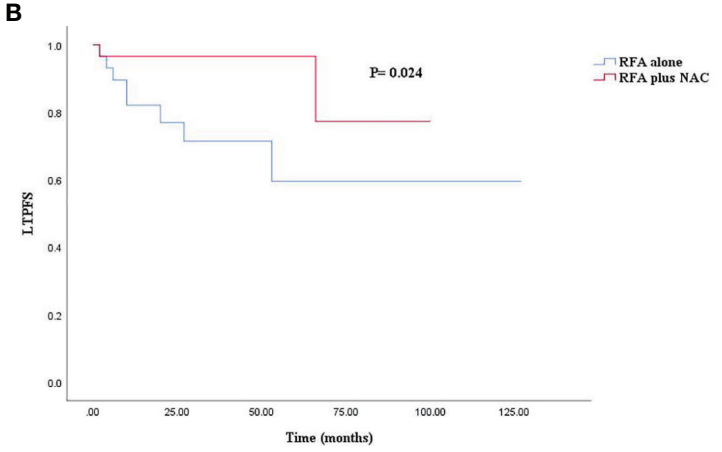

FIGURE 1 | Kaplan-Meier survival curve for LTFS of the radiofrequency ablation (RFA) alone and plus neoadjuvant chemotherapy (NAC) groups. (A) Unmatched analyses and (B) propensity-score-matched analyses.

research results was confirmed again by propensity score matching analysis. The analysis simulates the randomization of prospective studies and reduces the bias caused by confounding variables. To the best of our knowledge, this is the first comparative retrospective study to explore the prognostic advantage of RFA plus NAC. Previous single-arm studies were only for patients, who were initially unsuitable for local ablation treatment and were finally treated with RFA after conversion therapy $(25,26)$.

Nearly $50 \%$ of the basic clinical characteristics were significantly different between the two groups in this study, which might have an impact on the prognosis. Age $>60$ years, synchronous metastases, number of liver metastases $>2$, and modified ablation CRS $>2$ are considered as factors of poor prognosis for CRLM, while adjuvant chemotherapy after RFA is considered as a factor for protecting prognosis $(13,17)$. The RFA only group consisted of a high proportion of people $>60$ years old or who did not receive adjuvant chemotherapy after RFA. The plus NAC group also consisted of a high proportion of synchronous metastases, a number of liver metastases $>2$, and modified ablation CRS $>2$. Interestingly, after multivariate analysis of LTPFS, PFS, and OS, except for adjuvant chemotherapy after RFA, other bias factors did not have a critical impact on the prognosis. The effect of adjuvant chemotherapy after RFA on the prognosis could not be resolved in the retrospective study, and further prospective trials are essential.

The high LTP rate is an obstacle to the widespread use of RFA (27). The 3-year LTP rate of the RFA alone group was nearly three times higher than that in the plus NAC group. In the multivariate analysis of LTPFS, the use of NAC and the size of the lesion have a significant impact on LTPFS. Previous studies have confirmed that the size of the lesion affects the incidence of LTP after RFA in CRLM patients (28). The efficacy of RFA will reduce with the increase in lesion size $(27,29)$. The proportion of the largest diameter $>3 \mathrm{~cm}$ in the plus NAC group was higher, while the LTPFS was better than the RFA alone group. In this study, nearly half of the CRLM patients in the plus NAC group achieved an objective response, i.e., reduced lesion size, and associated improvement in LTPFS.

Only NAC was the single variable of significance in multivariate analysis of PFS. The improved PFS due to NAC reduced the lesion diameter and eliminated the micrometastatic sites, which ultimately reduced the local and distant recurrence

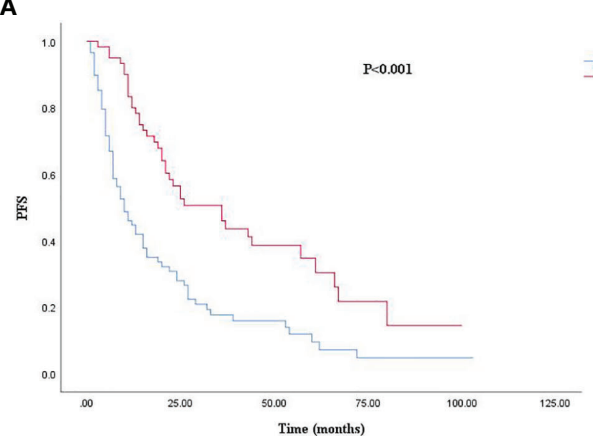

B

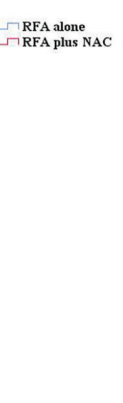

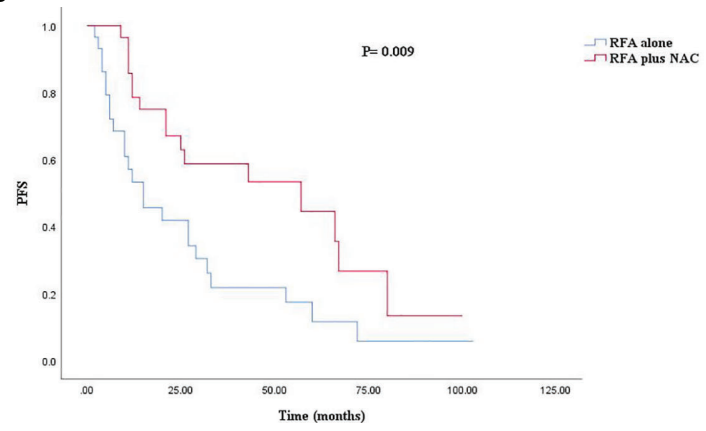

FIGURE 2 | Kaplan-Meier survival curve for PFS of the RFA alone and plus NAC groups. (A) Unmatched analyses and (B) propensity-score-matched analyses. 
A

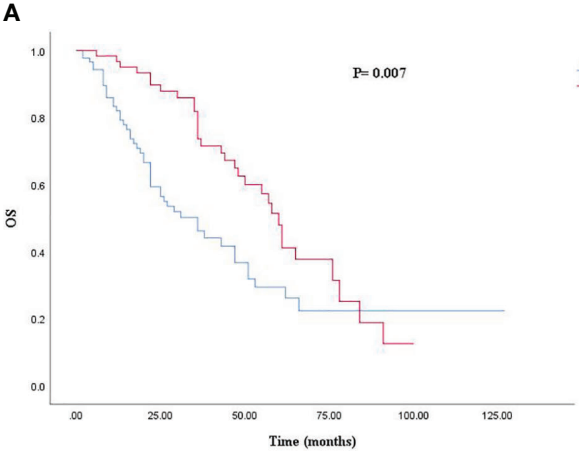

B

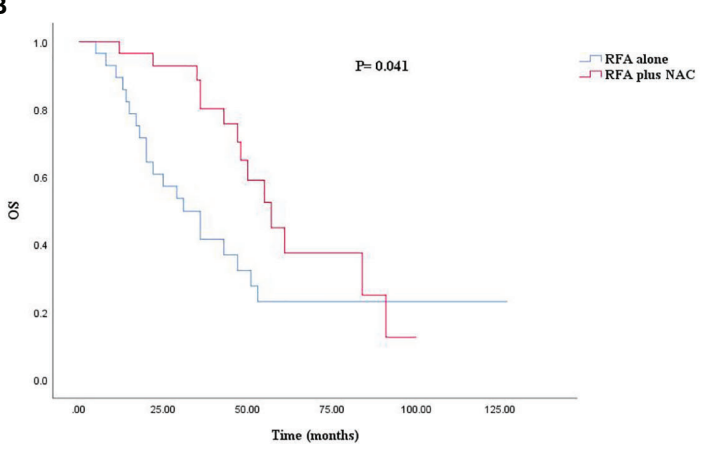

FIGURE 3 | Kaplan-Meier survival curve for OS of the RFA alone and plus NAC groups. (A) Unmatched analyses and (B) propensity-score-matched analyses.

TABLE 4 | Analysis of prognostic factors associated with LTPFS.

\begin{tabular}{|c|c|c|c|c|c|}
\hline Prognostic factor & $n$ & \multicolumn{2}{|c|}{ Univariate } & \multicolumn{2}{|c|}{ Multivariate } \\
\hline \multicolumn{6}{|l|}{ NAC } \\
\hline No & 88 & & & & \\
\hline Yes & 61 & $0.280(0.133-0.592)$ & 0.001 & $0.219(0.088-0.543)$ & 0.001 \\
\hline \multicolumn{6}{|l|}{ Gender } \\
\hline Female & 53 & & & & \\
\hline$\leq 60$ & 78 & & & & \\
\hline$>60$ & 71 & 1.509 (0.806-2.825) & 0.199 & & \\
\hline \multicolumn{6}{|c|}{ CEA at diagnosis (ng/ml) } \\
\hline$\leq 30$ & 116 & & & & \\
\hline$>30$ & 33 & 0.749 (0.331-1.693) & 0.487 & & \\
\hline \multicolumn{6}{|l|}{ Primary tumor } \\
\hline $\mathrm{T} 3 / \mathrm{T} 4$ & 132 & $1.118(0.398-3.145)$ & 0.832 & & \\
\hline \multicolumn{6}{|l|}{ LN metastasis } \\
\hline No & 35 & & & & \\
\hline Yes & 114 & $0.601(0.313-1.151)$ & 0.125 & & \\
\hline \multicolumn{6}{|l|}{ Timing of metastasis } \\
\hline Metachronous & 101 & & & & \\
\hline Synchronous & 48 & 0.509 (0.242-1.069) & 0.075 & 1.582 (0.669-3.739) & 0.296 \\
\hline \multicolumn{6}{|c|}{ Number of liver metastases } \\
\hline$<3$ & 122 & & & & \\
\hline$\geq 3$ & 27 & $1.030(0.474-2.238)$ & 0.941 & & \\
\hline \multicolumn{6}{|c|}{ Size of the largest lesion (cm) } \\
\hline$<3$ & 110 & & & & \\
\hline$\geq 3$ & 39 & $2.677(1.434-5.000)$ & 0.002 & 3.837 (1.973-7.463) & $<0.001$ \\
\hline
\end{tabular}

$H R$, hazard ratio; LN, lymph nodes; CEA, carcinoembryonic antigen.

${ }^{*}$ Node-positive primary tumor + disease-free interval from primary resection to the diagnosis of liver metastasis $<12$ months + more than one liver tumor + size of largest tumor $>3 \mathrm{~cm}+$ CEA level $>30 \mathrm{ng} / \mathrm{ml}$ ( $\mathrm{mg} / \mathrm{L})$. 
TABLE 5 | Analysis of prognostic factors associated with PFS.

\begin{tabular}{|c|c|c|c|c|c|}
\hline Prognostic factor & $n$ & \multicolumn{2}{|c|}{ Univariate } & \multicolumn{2}{|c|}{ Multivariate } \\
\hline \multicolumn{6}{|l|}{ NAC } \\
\hline Yes & 61 & $0.423(0.284-0.629)$ & $<0.001$ & $0.430(0.263-0.704)$ & 0.001 \\
\hline \multicolumn{6}{|l|}{ Gender } \\
\hline Female & 53 & & & & \\
\hline$\leq 60$ & 78 & & & & \\
\hline$>60$ & 71 & $1.693(1.151-2.491)$ & 0.007 & $1.438(0.961-2.154)$ & 0.078 \\
\hline \multicolumn{6}{|c|}{ CEA at diagnosis (ng/ml) } \\
\hline$\leq 30$ & 116 & & & & \\
\hline$>30$ & 33 & $1.354(0.889-2.062)$ & 0.158 & & \\
\hline \multicolumn{6}{|l|}{ Primary tumor } \\
\hline \multicolumn{6}{|l|}{ LN metastasis } \\
\hline No & 35 & & & & \\
\hline Yes & 114 & $1.129(0.722-1.765)$ & 0.596 & & \\
\hline \multicolumn{6}{|l|}{ Timing of metastasis } \\
\hline Metachronous & 101 & & & & \\
\hline Synchronous & 48 & $0.684(0.455-1.030)$ & 0.069 & 1.379 (0.815-2.332) & 0.231 \\
\hline \multicolumn{6}{|c|}{ Number of liver metastases } \\
\hline$<3$ & 122 & & & & \\
\hline$\geq 3$ & 27 & $0.956(0.588-1.554)$ & 0.855 & & \\
\hline \multicolumn{6}{|c|}{ Size of largest lesion $(\mathrm{cm})$} \\
\hline$<3$ & 110 & & & & \\
\hline$\geq 3$ & 39 & $1.126(0.732-1.732)$ & 0.588 & & \\
\hline
\end{tabular}

HR, hazard ratio; LN, lymph nodes; CEA, carcinoembryonic antigen.

${ }^{*}$ Node-positive primary tumor + disease-free interval from primary resection to the diagnosis of liver metastasis $<12$ months + more than one liver tumor + size of largest tumor $>3 \mathrm{~cm}+$ CEA level $>30 \mathrm{ng} / \mathrm{ml}(\mathrm{mg} / \mathrm{L})$.

rates. For resectable CRLM patients, surgical resection plus NAC could also improve PFS $(11,30)$.

In the multivariate analysis of OS, NAC, CEA, and adjuvant chemotherapy have a significant impact on OS. In CRLM patients undergoing RFA, poor survival prognosis is associated with high LTP (31). Also, as expected, NAC improves the prognosis by reducing local recurrence and distant metastasis. This result is more attractive than NAC in resectable CRLM. The high CEA is associated with poor OS. In 2016, Shady et al. proposed a modified ablation CRS score suitable for predicting the prognosis of OS and LTP for RFA, which was applied in the present study (17). Both the modified ablation CRS and the classic CRS assign CEA as one of items (32). Although postoperative adjuvant chemotherapy did not improve PFS and LTPFS, it improved the OS. CRLM patients require adjuvant chemotherapy after surgical resection, which is also recommended by the guidelines $(13,14)$. Herein, we proposed that adjuvant chemotherapy should also be routinely carried out after RFA, which could further reduce the risk of recurrence, necessitating prospective trials.

The postoperative complications in the plus NAC group were higher in this study. The use of NAC, followed by surgical resection, had higher complications than the surgery alone group (33). However, several studies demonstrated that these complications did not affect the prognosis of patients (34). A retrospective study included CRLM patients who underwent surgical resection from 1996 to 2006 and were divided into the NAC group and surgery alone group. The univariate and multivariate analyses showed that there was no difference between the two groups in terms of morbidity $(P=0.81)$, 
TABLE 6 | Analysis of prognostic factors associated with OS.

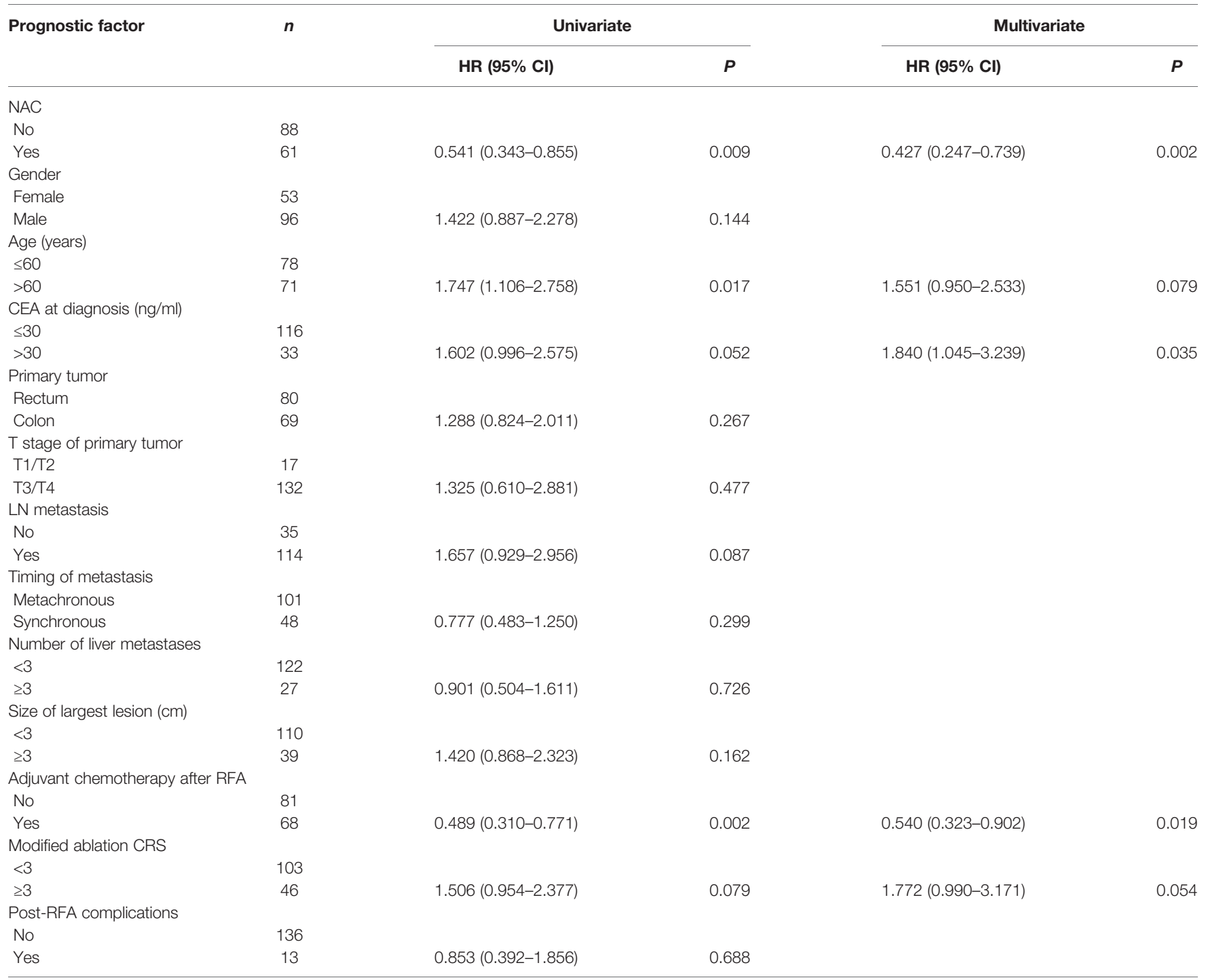

$H R$, hazard ratio; LN, Iymph nodes; CEA, carcinoembryonic antigen.

mortality $(P=0.29)$, PFS $(P=0.25)$, and OS $(P=0.30)$ (35). This phenomenon is consistent with the multivariate results of this study and the complications caused by NAC that will not affect the prognosis of patients.

The present study has some limitations. First, this is a retrospective single-center study. Differences were detected in the clinical baseline characteristics of the two groups. Although propensity score matching and multivariate analysis have resolved the possible deviations caused by clinical baseline imbalance, the reduced sample size after matching may affect the results. Second, the sample size of this study is small, which might affect the results. Third, there may have been patients who were technically "ablatable" and were given chemotherapy by their oncologist. However, follow-up imaging may have demonstrated progression or complete remission which ultimately resulted in no RFA. These patients cannot be included in the study due to limitations of retrospective study. Finally, RAS mutation status is an important prognostic tool for the determination of LTPFS and OS. Also, limited to the flaws of retrospective study, the authors do not include RAS mutation status in this study.

The results of this study are positive, which is hopeful for patients requiring local ablation. In the future, there are several aspects that need to be further elucidated. For example, 1) the interval between NAC and RFA should be set within a few weeks to ensure safety but not affect the treatment and 2) the optimal cycle of NAC and whether there are advantages to adding targeted drugs. In summary, NAC reduces LTP and prolongs PFS and OS in CRLM patients. Although NAC increases postoperative complications, it does not affect the long-term prognosis. The application of NAC before RFA deserves further evaluation as it is speculative based on the results of this study to imply that NAC before RFA will be the standard of care. 
However, the application of NAC before RFA needs further verification by prospective clinical trials. Therefore, large-sample prospective, double-blind controlled trials are required to substantiate the feasibility of NAC, followed by RFA.

\section{DATA AVAILABILITY STATEMENT}

The raw data supporting the conclusions of this article will be made available by the authors, without undue reservation.

\section{ETHICS STATEMENT}

The studies involving human participants were reviewed and approved by the Medical Ethics Committee of the Cancer
Hospital of the University of Chinese Academy of Sciences (Zhejiang Cancer Hospital). The patients/participants provided their written informed consent to participate in this study.

\section{AUTHOR CONTRIBUTIONS}

All authors contributed to the study conception and design. Material preparation and data collection were performed by $\mathrm{YC}$, JW, and LX. Data analysis was performed by YC, YH, HJ, YX, and FH. The first draft of the manuscript was written by YC. The review and editing of the article were performed by YZ and JW. All authors commented on previous versions of the manuscript. All authors contributed to the article and approved the submitted version.

\section{REFERENCES}

1. Siegel RL, Miller KD, Jemal A. Cancer Statistics, 2020. CA: Cancer J Clin (2020) 70(1):7-30. doi: 10.3322/caac.21590

2. Sung H, Ferlay J, Siegel RL, Laversanne M, Soerjomataram I, Jemal A, et al. Global Cancer Statistics 2020: GLOBOCAN Estimates of Incidence and Mortality Worldwide for 36 Cancers in 185 Countries. CA: Cancer J Clin (2021) 71(3):209-49. doi: 10.3322/caac.21660

3. Foster JH. Treatment of Metastatic Disease of the Liver: A Skeptic's View. Semin Liver Dis (1984) 4(2):170-9. doi: 10.1055/s-2008-1040656

4. Adam R, de Gramont A, Figueras J, Kokudo N, Kunstlinger F, Loyer E, et al. Managing Synchronous Liver Metastases From Colorectal Cancer: A Multidisciplinary International Consensus. Cancer Treat Rev (2015) 41 (9):729-41. doi: 10.1016/j.ctrv.2015.06.006

5. Norén A, Eriksson HG, Olsson LI. Selection for Surgery and Survival of Synchronous Colorectal Liver Metastases; a Nationwide Study. Eur J Cancer (Oxford England: 1990) (2016) 53:105-14. doi: 10.1016/j.ejca.2015.10.055

6. Angelsen JH, Horn A, Sorbye H, Eide GE, Loes IM, Viste A. Population-Based Study on Resection Rates and Survival in Patients With Colorectal Liver Metastasis in Norway. Br J Surg (2017) 104(5):580-9. doi: 10.1002/bjs.10457

7. Engstrand J, Nilsson H, Stromberg C, Jonas E, Freedman J. Colorectal Cancer Liver Metastases - A Population-Based Study on Incidence, Management and Survival. BMC Cancer (2018) 18(1):78. doi: 10.1186/s12885-017-3925-x

8. Meijerink MR, van den Tol MP, Puijk RS. The Rapidly Expanding Role of Thermal Ablation in the Treatment of Colorectal Liver Metastases. Hepatobiliary Surg Nutr (2020) 9(4):522-5. doi: 10.21037/hbsn.2019.11.22

9. Hao W, Binbin J, Wei Y, Kun Y. Can Radiofrequency Ablation Replace Liver Resection for Solitary Colorectal Liver Metastasis? A Systemic Review and Meta-Analysis. Front Oncol (2020) 10:561669. doi: 10.3389/fonc.2020.561669

10. Wong SL, Mangu PB, Choti MA, Crocenzi TS, Dodd GD3rd, Dorfman GS, et al. American Society of Clinical Oncology 2009 Clinical Evidence Review on Radiofrequency Ablation of Hepatic Metastases From Colorectal Cancer. J Clin Oncol (2010) 28(3):493-508. doi: 10.1200/JCO.2009.23.4450

11. Wang ZM, Chen YY, Chen FF, Wang SY, Xiong B. Peri-Operative Chemotherapy for Patients With Resectable Colorectal Hepatic Metastasis: A Meta-Analysis. Eur J Surg Oncol (2015) 41(9):1197-203. doi: 10.1016/ j.ejso.2015.05.020

12. Benson AB, Venook AP, Al-Hawary MM, Arain MA, Chen YJ, Ciombor KK, et al. Colon Cancer, Version 2.2021, NCCN Clinical Practice Guidelines in Oncology. J Natl Compr Canc Netw (2021) 19(3):329-59. doi: 10.6004/ jnccn.2021.0012

13. Van Cutsem E, Cervantes A, Adam R, Sobrero A, Van Krieken JH, Aderka D, et al. ESMO Consensus Guidelines for the Management of Patients With Metastatic Colorectal Cancer. Ann Oncol (2016) 27(8):1386-422. doi: 10.1093/annonc/mdw235

14. Diagnosis, Treatment Guidelines For Colorectal Cancer Working Group C. Chinese Society of Clinical Oncology (CSCO) Diagnosis and Treatment Guidelines for Colorectal Cancer 2018 (English Version). Chin J Cancer Res (2019) 31(1):117-34. doi: 10.21147/j.issn.1000-9604.2019.01.07

15. Gillams A, Goldberg N, Ahmed M, Bale R, Breen D, Callstrom M, et al. Thermal Ablation of Colorectal Liver Metastases: A Position Paper by an International Panel of Ablation Experts, The Interventional Oncology Sans Frontieres Meeting 2013. Eur Radiol (2015) 25(12):3438-54. doi: 10.1007/ s00330-015-3779-z

16. Kurilova I, Bendet A, Petre EN, Boas FE, Kaye E, Gonen M, et al. Factors Associated With Local Tumor Control and Complications After Thermal Ablation of Colorectal Cancer Liver Metastases: A 15-Year Retrospective Cohort Study. Clin Colorectal Cancer (2021) 20(2):e82-95. doi: 10.1016/ j.clcc.2020.09.005

17. Shady W, Petre EN, Gonen M, Erinjeri JP, Brown KT, Covey AM, et al. Percutaneous Radiofrequency Ablation of Colorectal Cancer Liver Metastases: Factors Affecting Outcomes-A 10-Year Experience at a Single Center. Radiology (2016) 278(2):601-11. doi: 10.1148/radiol.2015142489

18. Hof J, Wertenbroek MW, Peeters PM, Widder J, Sieders E, de Jong KP. Outcomes After Resection and/or Radiofrequency Ablation for Recurrence After Treatment of Colorectal Liver Metastases. Br J Surg (2016) 103(8):105562. doi: 10.1002/bjs.10162

19. Meijerink MR, Puijk RS, van Tilborg A, Henningsen KH, Fernandez LG, Neyt $\mathrm{M}$, et al. Radiofrequency and Microwave Ablation Compared to Systemic Chemotherapy and to Partial Hepatectomy in the Treatment of Colorectal Liver Metastases: A Systematic Review and Meta-Analysis. Cardiovasc Intervent Radiol (2018) 41(8):1189-204. doi: 10.1007/s00270-018-1959-3

20. Ruers T, Van Coevorden F, Punt CJ, Pierie JE, Borel-Rinkes I, Ledermann JA, et al. Local Treatment of Unresectable Colorectal Liver Metastases: Results of a Randomized Phase II Trial. J Natl Cancer Inst (2017) 109(9):djx015. doi: 10.1093/jnci/djx015

21. van Amerongen MJ, Jenniskens SFM, van den Boezem PB, Futterer JJ, de Wilt JHW. Radiofrequency Ablation Compared to Surgical Resection for Curative Treatment of Patients With Colorectal Liver Metastases - A Meta-Analysis. HPB (Oxford) (2017) 19(9):749-56. doi: 10.1016/j.hpb.2017.05.011

22. Takahashi H, Berber E. Role of Thermal Ablation in the Management of Colorectal Liver Metastasis. Hepatobiliary Surg Nutr (2020) 9(1):49-58. doi: 10.21037/hbsn.2019.06.08

23. Tanaka K, Adam R, Shimada H, Azoulay D, Levi F, Bismuth H. Role of Neoadjuvant Chemotherapy in the Treatment of Multiple Colorectal Metastases to the Liver. Br J Surg (2003) 90(8):963-9. doi: 10.1002/bjs.4160

24. Strowitzki MJ, Schmidt T, Keppler U, Ritter AS, Mahmoud S, Klose J, et al. Influence of Neoadjuvant Chemotherapy on Resection of Primary Colorectal Liver Metastases: A Propensity Score Analysis. J Surg Oncol (2017) 116 (2):149-58. doi: 10.1002/jso.24631 
25. Nielsen K, Scheffer HJ, Volders JH, van der Vorst MJ, van Tilborg AA, Comans EF, et al. Radiofrequency Ablation to Improve Survival After Conversion Chemotherapy for Colorectal Liver Metastases. World J Surg (2016) 40(8):1951-8. doi: 10.1007/s00268-016-3554-6

26. Knudsen AR, Kannerup AS, Mortensen FV, Nielsen DT. Radiofrequency Ablation of Colorectal Liver Metastases Downstaged by Chemotherapy. Acta Radiol (2009) 50(7):716-21. doi: 10.1080/02841850902991634

27. Han K, Kim JH, Yang SG, Park SH, Choi HK, Chun SY, et al. A Single-Center Retrospective Analysis of Periprocedural Variables Affecting Local Tumor Progression After Radiofrequency Ablation of Colorectal Cancer Liver Metastases. Radiology (2021) 298(1):212-8. doi: 10.1148/radiol.2020200109

28. Nieuwenhuizen S, Puijk RS, van den Bemd B, Aldrighetti L, Arntz M, van den Boezem PB, et al. Resectability and Ablatability Criteria for the Treatment of Liver Only Colorectal Metastases: Multidisciplinary Consensus Document From the COLLISION Trial Group. Cancers (Basel) (2020) 12(7):1779. doi: 10.3390/cancers12071779

29. Sotirchos VS, Petrovic LM, Gonen M, Klimstra DS, Do RK, Petre EN, et al. Colorectal Cancer Liver Metastases: Biopsy of the Ablation Zone and Margins Can Be Used to Predict Oncologic Outcome. Radiology (2016) 280(3):949-59. doi: 10.1148/radiol.2016151005

30. Ke S, Zhan S, Zhu H, Yan D. Topics Related to Neoadjuvant Chemotherapy for Resectable Liver Metastases From Colorectal Cancer.J BUON (2018) 23(2):296-301.

31. Solbiati L, Ahmed M, Cova L, Ierace T, Brioschi M, Goldberg SN. Small Liver Colorectal Metastases Treated With Percutaneous Radiofrequency Ablation: Local Response Rate and Long-Term Survival With Up to 10-Year Follow-Up. Radiology (2012) 265(3):958-68. doi: 10.1148/radiol.12111851

32. Fong Y, Fortner J, Sun RL, Brennan MF, Blumgart LH. Clinical Score for Predicting Recurrence After Hepatic Resection for Metastatic Colorectal Cancer: Analysis of 1001 Consecutive Cases. Ann Surg (1999) 230(3):30918; discussion 18-21. doi: 10.1097/00000658-199909000-00004

33. Ribeiro HS, Costa WLJr., Diniz AL, Godoy AL, Herman P, Coudry RA, et al. Extended Preoperative Chemotherapy, Extent of Liver Resection and Blood
Transfusion are Predictive Factors of Liver Failure Following Resection of Colorectal Liver Metastasis. Eur J Surg Oncol (2013) 39(4):380-5. doi: 10.1016/j.ejso.2012.12.020

34. Vigano L, Capussotti L, De Rosa G, De Saussure WO, Mentha G, RubbiaBrandt L. Liver Resection for Colorectal Metastases After Chemotherapy: Impact of Chemotherapy-Related Liver Injuries, Pathological Tumor Response, and Micrometastases on Long-Term Survival. Ann Surg (2013) 258(5):731-40; discussion 41-2. doi: 10.1097/SLA.0b013e $3182 \mathrm{a} 6183 \mathrm{e}$

35. Scoggins CR, Campbell ML, Landry CS, Slomiany BA, Woodall CE, McMasters KM, et al. Preoperative Chemotherapy Does Not Increase Morbidity or Mortality of Hepatic Resection for Colorectal Cancer Metastases. Ann Surg Oncol (2009) 16(1):35-41. doi: 10.1245/s10434008-0190-x

Conflict of Interest: The authors declare that the research was conducted in the absence of any commercial or financial relationships that could be construed as a potential conflict of interest.

Publisher's Note: All claims expressed in this article are solely those of the authors and do not necessarily represent those of their affiliated organizations, or those of the publisher, the editors and the reviewers. Any product that may be evaluated in this article, or claim that may be made by its manufacturer, is not guaranteed or endorsed by the publisher.

Copyright $\odot 2021$ Chen, $X u, X u$, Han, Huang, Jiang, Wu and Zhang. This is an openaccess article distributed under the terms of the Creative Commons Attribution License (CC BY). The use, distribution or reproduction in other forums is permitted, provided the original author(s) and the copyright owner(s) are credited and that the original publication in this journal is cited, in accordance with accepted academic practice. No use, distribution or reproduction is permitted which does not comply with these terms. 